\title{
Inicios de las Boys Town en España: la Ciudad de los Muchachos de Vallecas (Madrid)
}

\author{
Francisco Javier Pericacho Gómez \\ Mario Andrés-Candelas²
}

\section{Resumen}

A principios del siglo XX surgen en Estados Unidos una serie de experiencias denominadas Boys Town destinadas a niños y adolescentes en situación de exclusión social. Este tipo de iniciativas llegó a España 40 años más tarde y se denominó Ciudades de los Muchachos. Esta investigación estudia la experiencia pedagógica realizada en la Ciudad de los Muchachos de Vallecas, fundada en 1947, durante sus primeros años de funcionamiento. Para realizar este análisis se han utilizado fuentes inéditas, como son los documentos existentes en el Fondo Romero Marín, conservado en el Museo de Historia de la Educación M. B. Cossío de la Facultad de Educación (Universidad Complutense de Madrid), que cuenta con aproximadamente 895 trabajos o memorias de fin de curso que realizaron los estudiantes de la Facultad de Filosofía y Letras, pertenecientes a la sección de Pedagogía, entre 1950 y 1975. Los estudiantes sistematizaron sus observaciones de las prácticas escolares realizadas en distintos ámbitos de la enseñanza, entre los que se encuentra la Ciudad de los Muchachos de Vallecas. A través del estudio de estas memorias y de bibliografía académica destacada, prensa de la época y otros materiales y documentos relevantes, se expone la implementación y los primeros años de funcionamiento en España del modelo internacional de las Boys Town. El estudio de la naturaleza de esta emblemática experiencia aún activa en la actualidad, su innovadora propuesta socio-educativa y las particulares características del lugar y del momento histórico en el que surgió, evidencia un gran valor histórico y pedagógico.

\section{Palabras clave}

Ciudad de los Muchachos - Historia de la educación - Exclusión social - Infancia Vallecas (Madrid).

\section{Beginning of Boys Town in Spain: The Ciudad de los Muchachos of Vallecas (Madrid)}

\section{Abstract}

A series of educational experiences, known as The Boys Town, were set up for children and teenagers at risk of social exclusion in the United States in the early 20th

\footnotetext{
1- Universidad Antonio de Nebrija, España. Contacto: pericacho.javier@yahoo.es

2 - Universidad Complutense de Madrid, España. Contacto: mario.andres.candelas@gmail.com

DOl: http://dx.doi.org/10.1590/S1678-4634201709163028
} 
century. These initiatives appeared in Spain 40 years later, under the name of Ciudades de los Muchachos. This investigation is about the pedagogical experience carried out at the Ciudad de los Muchachos in Vallecas during its first year of operation, around 1947. For the analysis, we have used unpublished sources, such as documents from the Romero Martin Fund, preserved in the Museum of History of Education M.B. Cossio of the Faculty of Education (Complutense University of Madrid). This fund has approximately 895 academic works or end-of-course reports written by students of the Faculty of Philosophy and Literature, section of Pedagogy, between 1950 and 1975. Students systematized their observations during internships occurring in different types of schools, among which was the Ciudad de los Muchachos in Vallecas. Through the study of these reports and academic literature, newspapers at the time and other relevant materials and documents, we look at the early years of the implementation of the international model of the Boys Town in Spain. The investigation of this iconic experience, which is still active today, its innovative socio-educational proposal and the special characteristics of the site and the historical moment in which appeared, reveals its great historical and educational value.

\section{Keywords}

Boys Town - History of education - Social education - Childhood - Vallecas (Madrid).

\section{Introducción}

A finales del siglo XIX y principios del siglo XX la infancia empieza a tomar relevancia social, saliendo de la invisibilidad y ocupando su lugar en el espacio público. El proceso de construcción de este espacio es una cuestión que aún hoy está en discusión, ya que existen diferentes tesis al respecto. La tesis que más reconocimiento ha tenido ha sido la defendida por Ariès (1987), quien sostiene que es a partir de los siglos XVI y XVII cuando comienzan a surgir los sentimientos específicos hacia la infancia. Estos sentimientos son el mimoseo, surgido en el seno de la familia, y la preocupación moral y psicológica, surgido entre los moralistas y educadores. La aparición y consolidación de estos sentimientos hicieron surgir el espacio social de la infancia moderna, dando lugar a su descubrimiento o invención que se consolidó a lo largo del siglo XVIII, influido por la Ilustración y la burguesía.

Sin embargo, para DeMause (1982), otro autor fundamental en este campo, la tesis de Ariès es insostenible, ya que las evidencias muestran como desde la antigüedad existe el espacio de la infancia, pero el trato y la consideración que esta ha venido recibiendo han ido evolucionando, desde el infanticidio y el abandono, hasta la socialización y la ayuda. En resumen, para DeMause, "la historia de la infancia es una pesadilla de la que hemos empezado a despertar hace muy poco" (1982, p. 15). A pesar de estas diferencias en el proceso, ambas corrientes coinciden en que en los inicios del siglo XX el espacio social 
de la infancia comienza a sufrir grandes transformaciones. Tanto es así, que el siglo XX, gracias al título de la obra de la autora sueca Ellen Key (1906), que tuvo una gran difusión y repercusión en España, comenzó conociéndose como El siglo de los niños. Es evidente que podríamos discutir profundamente sobre el alcance de este título y sus implicaciones, pero es cierto que a lo largo del siglo XX surgió una gran cantidad de instituciones que pusieron su mirada en la infancia, y en especial, en la infancia excluida o peligrosa (ANDRÉS-CANDELAS, 2016).

Esto es así ya que la infancia era, y en cierta medida todavía es hoy, objeto de preocupación en dos sentidos muy opuestos, por un lado, se considera que los niños y niñas están en peligro y deben ser protegidos, y por otro, la sociedad se debe proteger de algunas de estas niñas y niños peligrosos (CARREÑO, 2005; RODRIGUES-BREITMAN, 1994), en palabras de Morrow (2005), existe preocupación por los problemas de los niños y de las niñas y también por las niñas y niños problemáticos. Para este segundo grupo de niños y niñas van naciendo diferentes dispositivos con muy diferentes orientaciones. En este sentido se logran distinguir dos tendencias, los llamados child savers y los kiddie libbers (MELTON, 2005). Los primeros centrados en un enfoque de protección, que convierte en objetos a salvaguardar a los niños y niñas, y el segundo centrado en un enfoque de derechos, con una visión que otorga más participación y protagonismo a los propios niños y niñas. Si bien es cierto que algunos autores posteriormente han intentado buscar la reconciliación entre ambos enfoques (CASCARDI et al., 2015), en la época que nos ocupa, esto es, los inicios del siglo XX, las instituciones que se crearon bajo la impronta de estos diferentes enfoques tuvieron consecuencias diferentes para los niños y niñas. Sirva como ejemplo el excelente análisis que realiza Platt (1988) sobre la creación de los tribunales penales para niños y niñas en Estados Unidos, que a pesar de nacer de intereses inicialmente bienintencionados que pretendían salvar a los niños y niñas excluidos, lograron el efecto contrario, incluyendo la privación de algunos de sus derechos más básicos.

Desde propuestas más cercanas a la visión de los kiddy libbers, ya que buscaban la autonomía a través del reconocimiento de cierto protagonismo a los niños y niñas excluidos en su propio proceso educativo, surgen a principios del siglo XX en Estados Unidos una serie de experiencias denominadas Boys Town que, en España, cuando llegaron 40 años más tarde, pasaron a denominarse Ciudades de los Muchachos. Estas iniciativas de carácter socioeducativo y resocializador nacen bajo la influencia y dirección de diferentes hermandades religiosas.

Este artículo se centra en una de estas experiencias concretas, la Ciudad de los Muchachos de Vallecas, situada en el sureste de la ciudad de Madrid (España). Desde una perspectiva de innovación pedagógica y fuerte compromiso socio-educativo, constituye una de las iniciativas más significativas y longevas en el territorio español, en funcionamiento desde mediados del silgo XX (PERICACHO, 2014a). En el presente trabajo, tras la constatación de la reducida producción de estudios que muestren la realidad de los primeros años de esta institución, pretendemos estudiarla a través del estudio de documentación relevante, bibliografía académica destacada, prensa de la época y el análisis de fuentes inéditas de un gran valor histórico: las memorias realizadas por 
los estudiantes de la sección de Pedagogía de la Facultad de Filosofía y Letras de la Universidad Complutense de Madrid entre 1950 y 1975, documentos pertenecientes al fondo documental Romero Marín, conservado en el Museo de Historia de la Educación Manuel Bartolomé Cossío de la Facultad de Educación de la misma universidad. Tras el estudio exhaustivo de los más de 895 trabajos o memorias de fin de curso, se seleccionaron aquellos trabajos que tenían como objetivo central de estudio la Ciudad de los Muchachos y como marco geográfico y temporal de observación Vallecas (Madrid) entre 1950 y 1967 por ser los primeros años de funcionamiento.

Por tanto, se pretende contestar los siguientes interrogantes: ¿dónde se encuentran sus principales influencias y antecedentes pedagógicos?, ¿en qué momento y contexto histórico se implementa?, ¿cómo se desarrolló su nacimiento, consolidación y labor socioeducativa?, y, por último, ¿cuál era su estructura inicial?

En coherencia, ante la extensión del tema y para su mejor profundización, sólo nos centramos en estos puntos y en un periodo histórico, los primeros años. Por tanto, no agotamos todos los posibles temas de análisis que se suscitan.

\section{Boys town: influencias, antecedentes y evolución histórica}

Las Boys Town o Ciudades de los Muchachos se definen como instituciones estructuradas y comunidades socio-educativas. Su prioridad principal consiste en la formación de niños y adolescentes en situación de exclusión social a través de su formación académica, laboral y moral. Esta labor se realiza en un contexto generalmente alejado de los grandes núcleos urbanos. Por tanto, su objetivo fundamental es la reinserción socio-educativa de los jóvenes. Para tal fin, los procesos cotidianos se encuentran regidos desde una auto-regulación responsable, participativa y activa de todos los niños y jóvenes, unos patrones que, al menos en sus orígenes, se basan en el autogobierno (FERNÁNDEZ, 2009). La asistencia a este tipo de centros puede ser en régimen de internado o como alumnos externos.

Las premisas fundamentales quedan resumidas en tres puntos: una base filosófica y educativa que concibe que cualquier niño puede salir de su situación de exclusión, un elevado autogobierno que vertebra la cotidianidad del centro, y por último, una convivencia marcada por un gran sentido socio-educativo. Teniendo en cuenta estas premisas, se puede considerar que la principal influencia educativa subyace en las teorías de Rousseau (1982) sobre la bondad natural del ser humano frente a las influencias corruptoras de la sociedad, ideas reflejadas en su libro Emilio o de la Educación. El joven Emilio es formado en la convivencia con la naturaleza, alejado y resguardado de las coerciones sociales. Asimismo, se constata una amplia influencia en las ideas de Dewey (1995) sobre la conversión del entorno escolar en una comunidad educativa sin apenas intervención de los adultos, así como su noción de construcción de comunidad y democracia, recogidas en su obra Democracia y educación. Igualmente se puede observar una gran influencia en las ideas de Kerschensteiner (RÖHRS, 1993) sobre la formación profesional y su idea del trabajo como fuente que devuelve al hombre a la comunidad.

En el plano práctico existieron varias iniciativas que influyeron en la metodología de las Ciudades de los Muchachos, algunas de las más significativas las encontramos 
en las Repúblicas juveniles que se extendieron por el continente Europeo y Americano, popularizadas por Regina Lago (1931) en España; en el orfanato de Cempuis del profesor francés Paul Robin en 1880 (CUEVAS, 2003); y por último, en la Colonia Gorki de Makarenko en 1920 (TRILLA, 1985), experiencia que disfrutó de una elevada difusión gracias a su ampliamente difundido Poema pedagógico (MAKARENKO, 1996).

Las Boys Town adquieren su mayor difusión a través del sacerdote de la Iglesia Católica Edward Joseph Flanagan (1886-1948) y su creación de la primera Boys Town en 1926 (Nebraska, EEUU), en la que participaban niños con edades comprendidas entre los 10 y los 16 años (REILLY; WARNEKE, 2008). En esta experiencia, cuya premisa fundamental consistía en fomentar la responsabilidad entre los muchachos en situación de abandono o exclusión social por medio del autogobierno (FERNÁNDEZ, 2009), existían cargos ocupados por los alumnos que vigilaban el cumplimiento de la normativa, siendo estos los que ayudaban a los recién llegados y juzgaban los problemas cotidianos.

Debido a esta iniciativa y, en buena medida a través de la exitosa película Boys Town, conocida en España como Forja de hombres y en Brasil como Com os Braços Abertos, estrenada en 1938 con Spencer Tracy encarnando al Padre Flanagan y Mickey Rooney como chico con problemas (película premiada con dos Oscar al mejor actor y mejor guión original), la experiencia se populariza, extendiéndose e implementándose en varios países. Así, Fernández (2009) señala lo siguiente: durante 1945 en Italia se crearon veinte pueblos de niños entre los que destacan el Poblado de los muchachos del Padre Rivolta en Chivitavecchia, en 1949 en Argentina se crea por Eva Perón la Ciudad Infantil, en Francia Les Rayons de Soleil y en Hungría el Pueblo de los niños, entre otros. En la actualidad EEUU alberga varias Boys Town repartidas en doce áreas por todo el país.

La innovadora propuesta teórica y práctica de este tipo de iniciativas no tardó mucho en llegar a España. Así, se fundaron varias Ciudades de los Muchachos en Orense (FROUFE QUINTAS, 1999), Valencia (PAYÁ, 1950) y Madrid. En este último caso concreto, existen dos experiencias que siguen en funcionamiento: la Ciudad de los Muchachos de Vallecas, origen de este estudio y del que nos ocuparemos en profundidad más adelante, y la Ciudad Escuela Muchachos de Leganés.

La Ciudad Escuela Muchachos de Leganés (CEMU) situada en la Avenida Doctor Fleming, en el municipio de Leganés, al sur de la Comunidad de Madrid, fue fundada en el año 1970 por el arquitecto Alberto Muñiz Sánchez, popularmente conocido como tío Alberto. Es una comunidad concebida por y para la educación integral de los niños en desventaja y/o conflicto social. Es una institución por la que han pasado más de tres mil niños y jóvenes. En la actualidad la ciudadanía infantil y juvenil se diferencia en alumnos externos y residentes. Las vías de ingreso son dos: bien por petición de los técnicos de servicios sociales que tienen la guarda o tutela de los niños, bien por petición expresa de los padres y madres que por diferentes motivos buscan un recurso residencial para sus hijos. En definitiva, es una ciudad-educadora que actúa con los mecanismos propios de un núcleo familiar, de esta forma, está provista de ayuntamiento, residencias, colegio, iglesia, zonas verdes, albergue, radio, zonas recreativas, granja, tiendas, huerto, talleres, etc. Su objetivo es el de restituir en los niños (ciudadanos) el medio convivencial apropiado para restaurar los daños y deficiencias de su pasado, trabajar su presente y orientar su futuro. 


\section{Posguerra y exclusión: contexto histórico y social en el barrio de Vallecas}

El 17 de julio de 1936 comienza la Guerra Civil Española, finalizando el 1 de abril de 1939. Se inicia al levantarse en armas una parte del ejército español asentado en Marruecos (dirigido por el general Francisco Franco) contra el gobierno republicano democráticamente elegido (presidido por Manuel Azaña). Tras la victoria del bando sublevado se inicia en España una dictadura de casi cuarenta años (1939-1975).

La Guerra Civil ha sido suficientemente tratada y estudiada en todo tipo de trabajos (OSSORIO, 1941; MAURÍN, 1966; AZAÑA, 1986; PRIETO, 1989; SAZ, 2004; TAGÚEÑA, 2005; KOLTSOV, 2009). Pese a las diferentes interpretaciones políticas que conlleva este episodio en la historia española (fácilmente apreciable en los diferentes estudios realizados), es unánime una afirmación común: el final de la guerra no acabó con la represión y la violencia, tampoco se generó una posible pacificación y reconciliación nacional. Por el contrario, el nuevo sistema político iniciado en 1939 se gestó desde una frontal división entre vencedores y vencidos. De esta forma, el periodo de posguerra se caracterizó por ser una larga etapa de autarquía económica, grandes carencias, hambre, aislamiento, supresión de libertades, dura represión política, analfabetismo y falta de servicios sociales.

Concretamente en Madrid, en este periodo histórico, se forman grandes suburbios de obreros fabriles (provenientes de zonas rurales) huyendo de la miseria del extrarradio y en búsqueda de un empleo, una gran corriente de movimientos migratorios hacia la capital madrileña (ALONSO-CORTES, 1966). Uno de aquellos suburbios y núcleos chabolistas es Puente de Vallecas, zona situada en el extremo Sur-Este de Madrid que sufrió circunstancias especialmente dramáticas durante la Guerra Civil Española debido a sus connotaciones políticas ligadas a la izquierda.

\footnotetext{
El barrio obrero del Puente pronto adquirió unas connotaciones izquierdistas, muy radicalizadas, con un destacado (y lúgubre) papel durante la guerra civil, por estar en la zona roja y su proximidad al frente del Jarama. Los atroces acontecimientos acaecidos (fusilamiento del alcalde republicano Amós Acero, quema de iglesias y ermitas, asesinato de todos los pasajeros de un tren, bombardeos que destruyen ambas poblaciones...) han provocado un bloqueo mental o pacto de silencio entre los habitantes que aún hoy se niegan a relatar nada de aquellos años. A esto sucedió una durísima posguerra en la que no faltaron las represalias personales y colectivas como la demolición del edificio del ayuntamiento. (FERNÁNDEZ, 2007, p. 57).
}

Los inmigrantes que fueron llegando no disponían de los recursos económicos suficientes para acceder a una vivienda, por lo que se fueron generando núcleos de chabolas en condiciones muy precarias, plantadas en muchas ocasiones en medio del barro sin ninguna condición mínima de habitabilidad (BUENO, 1963). Es importante señalar que entre 1950 y 1960 la población se multiplicó en Vallecas por 2,5, suponiendo un incremento anual de 17.354 personas, en 1966 sobrepasaba los 300.000 habitantes, con un 38,1\% de población activa y un 61,9 \% de población inactiva (ALONSO-CORTÉS, 1966). 
De esta forma, poco a poco se va a ir generando una particular leyenda negra de miseria, delincuencia y marginalidad que invade el imaginario social sobre Vallecas. Tal como se podía leer en la prensa de la época: "Inicióse la obra en el lugar más mísero de Madrid, el clásico barrio de las latas del Puente de Vallecas” (LA VANGUARDIA, 1951, p. 10). Algo que, por otra parte, discurre paralelo a una excepcional dinámica socio-cultural marcada por la multiplicación de iniciativas organizativas y una inmensa fuerza solidaria de apoyos de curas obreros, abogados laboralistas y partidos políticos clandestinos (FERNÁNDEZ, 2007).

Cabe destacar también que a principios del siglo XX el porcentaje de analfabetismo era del 56 \% y España ofrecía, junto con Portugal, Italia, Grecia, Rusia y los países de la Europa del Este, los porcentajes de analfabetismo más elevados del continente europeo (VIÑAO, 2009). Concretamente en Vallecas el analfabetismo era especialmente alto, los niños eran enviados a trabajar muy pronto (repartir recados, servir de aguadores, etc.). Según Bueno (1963), en el curso 1962 existía un 20 \% de hombres y un 40\% de mujeres que no sabían leer y escribir, asimismo existía un alto número de población infantil con edades comprendidas entre los 4 y los 8 años que no se cubría con los establecimientos educativos, existiendo siempre niños en espera para ingresar en los colegios. Tal como señala Alonso-Cortes (1966), la mayoría de los niños abandonaba la escuela al terminar su periodo de escolaridad obligatoria a los 14 años.

\section{Formación, implantación y composición del proyecto socio-educativo}

En el año 1940 llega a uno de los barrios obreros más deprimidos de Madrid (Puente de Vallecas) la primera comunidad religiosa de Agustinos Asuncionistas con el objetivo de atender a la juventud obrera, fundando la Parroquia del Dulce nombre de María. La idea de la Ciudad de los Muchachos nace debido a varios años de labor pastoral y educativa en el suburbio de Vallecas. Es en 1947 y dirigido por el Sacerdote Luis Madina Michelena (en adelante Padre Madina), cuando la comunidad religiosa, además de continuar con las labores propias de la Parroquia, comienza el proyecto de la Ciudad de los Muchachos, situada en la calle Manco de Lepanto, $\mathrm{n}^{\circ} 2$, enclavada dentro del barrio denominado Doña Carlota, perteneciente al municipio y distrito de Vallecas de Madrid.

A partir de ese año (1947) la comunidad de Agustinos Asuncionistas se encuentra ante el hecho de la incapacidad de las familias a la hora de atender las necesidades de los niños y niñas, los padres trabajaban todo el día y las madres hacían lo mismo para completar la economía doméstica como asistentas en el centro de Madrid. Los niños y niñas, por tanto, quedaban únicamente al cuidado de las vecinas y de los hermanos y hermanas mayores, que se veían en la obligación de dejar la escuela. Muchos de los adolescentes comenzaron a desarrollar actividades y conductas no cívicas, bien buscando en los basureros, bien organizando bandas (RUTE, 1961).

Por tanto, tras entender que en estos niños se encontraba el futuro, además de continuar con las labores de la Parroquia, se abre la guardería Infantil y la Escuela de 
Orientación Profesional. Esto se hace durante los años comprendidos entre 1948 y 1953, bajo la pretensión de regenerar a los mismos y al barrio. Así, durante estos años:

- Se inician y perfeccionan talleres de mecánica, carpintería, electricidad, oficina e imprenta y dibujo industrial.

- Se crea la guardería en un pequeño chalet que (debido a la buena aceptación) se amplía en 1952 construyendo una segunda planta y una residencia para las monjas.

Tras esto, tal como detalla Ávila (1962), se construye un nuevo pabellón de tres plantas en el que tenían cabida 350 niños. En este pabellón se encontraba la capilla, el salón de actos, el cuarto de antiguos alumnos, cuartos para la dirección y las clases donde recibían enseñanza primaria los niños que habiendo salido de la maternal, no tenían todavía edad para entrar en la escuela Profesional. De esta forma se consigue que todo el periodo de formación, desde sus primeros meses hasta los 14 ó 16 años, se desarrollara dentro de las Ciudad de los Muchachos. En principio la implantación de la Ciudad de los Muchachos disfrutó de bastante aceptación, tal y como señala Ávila (1962), prueba de ello era que desde hacía tres años se habían rechazado a más de 300 chicos que no tenían plaza.

El programa de estudios que se desarrollaba y los métodos de enseñanza que se practicaban estaban de acuerdo a la ideología religiosa propia de la comunidad Asuncionista. Tal y como se observa en las memorias, la finalidad principal que se pretendía era dar cobijo al niño desde su infancia hasta su llegada al servicio militar, enseñándole una profesión, educándole intelectualmente e inculcándole los principios de la moral cristiana, en un medio aislado del ambiente deprimido que le rodeaba (PÉREZ, 1953).

En definitiva, los objetivos que se pretendía alcanzar quedan resumidos en tres:

1) Proporcionar al niño o niña una sólida orientación y formación intelectual y moral.

2) Abstraer al niño del ambiente negativo que le rodeaba y que se entendía neutralizaría la labor pedagógica.

3) Preparar al niño para la vida.

\section{Relación con el régimen Franquista}

Como se ha señalado, el 17 de julio de 1936 comienza la Guerra Civil Española. Finalizado el conflicto bélico en 1939 se inicia en España una dictadura de casi cuarenta años (1939-1975). El cambio de signo político que dio comienzo con el franquismo generó no pocos cambios en el terreno educativo. Numerosas instituciones y organismos que habían promovido (con mayor o menor intensidad) el ideario político y educativo de la Segunda República (1931-1936) fueron acusados de fomentar intereses contrarios a la patria y, como consecuencia, obligados a cerrar sus puertas o modificar sus señas de identidad de acuerdo al nuevo modelo de hombre, sociedad y Estado que se pretendía implementar en el régimen. Sirva de ejemplo el Decreto de 17 de mayo de 1940 (ESPAÑA, 1940) por el que se ilegaliza a la emblemática Institución Libre de Enseñanza, se incautan sus bienes y se adscriben al Ministerio de Educación Nacional. 
La implementación y los primeros años de la Ciudad de los Muchachos se desarrollaron en este largo y conflictivo periodo histórico de depuración, prohibición y entorpecimiento a cualquier idea o práctica pedagógica que fuera diferente a los ideales del régimen político y pedagógico (PERICACHO, 2014b). Sin embargo, la relación del Régimen Franquista con la Ciudad de los Muchachos no parece haber sido uniforme. En un principio y tal como se advierte en las memorias, se observa simpatía con el proyecto, cuestión que se aprecia en Pérez (1953) al señalar una dedicatoria en una fotografía obsequiada al Padre Madina, en la que se leía: "Incansable apóstol de la Parroquia del Dulce Nombre de María y Director de la Ciudad de los Muchachos con mi simpatía y gratitud a su obra ejemplar, afectuosamente. Francisco Franco". La prensa de la época igualmente sirve de indicador:

Hace tres años, la prensa madrileña dedicó artículos y reportajes a la entonces naciente Ciudad de los Muchachos. Desde entonces desarrolla una labor silenciosa, pero eficaz. La semilla ha ido creciendo. No es todavía el árbol frondoso... más sus tiernas ramas cobijan centenares de inocentes criaturas (MADINA, 1950, p. 33).

Sin embargo, con el paso del tiempo, la relación se fue enfriando a medida que el compromiso con las necesidades y demandas de los vecinos por parte de la Ciudad de los Muchachos se profundizaba, por lo que será objeto de represalias del Régimen. Así, surgen conflictos de una elevada tensión política como la suspensión en 1972 de la IV Asamblea Cristiana de Vallecas que pretendía celebrarse en la Ciudad de los Muchachos o el asesinato de un alumno en una manifestación.

\section{Estructura de la Ciudad de los Muchachos de Vallecas}

A continuación, se muestran algunas pinceladas histórico-educativas sobre la estructura básica de esta institución, centrándonos principalmente en la Guardería, la Escuela de Orientación profesional, la interesante iniciativa de Autogobierno participativo y la Revista (como principal órgano de difusión y recaudación económica).

\section{La Guardería}

En 1950 se construyó la Guardería, algo de urgente necesidad ya que los padres y madres trabajaban todo el día fuera y no podían estar con sus hijos. Comenzó con veinte niños, en 1962 Ávila (1962) señala que había 150 niños. A su cargo estaban las hijas de la Caridad apoyadas por las Luisas de Marillac. Pasados unos años y tras las aportaciones económicas de varios madrileños, se transformó en un edificio de 2 plantas.

Secciones de la Guardería:

1) Las cunas, con niños entre los 15 meses y los 2 años.

2) La maternal, con niños de 2 años a 4 años.

3) Párvula, con niños de 4 a 8 años. 
En la misma también se realizaban conferencias religiosas y educativas enfocadas a la formación de madres, prácticamente todas las semanas había algún acto en este sentido, existiendo también conferencias extraordinarias con motivo de alguna fiesta (día de la Madre, Navidad, Reyes).

\section{La escuela de orientación profesional}

Las obras comenzaron en 1947 sobre un terreno de labranza llamado Terreno de los Frailes de San Martín, en el momento de construir el lugar era un estercolero que se pudo conseguir a precio reducido (lugar que al ir cavando para la construcción, se fueron dando cuenta de lo inmejorable del sitio). El periodo de iniciación duró 4 años, se construyó primeramente un salón de actos dedicado a conferencias y representaciones de teatro, con el fin de atraer a los hombres y por medio de ellos a sus hijos. A través del salón de actos y un bar que se instaló en él, se consiguió que algunos hombres se acercaran con deseos "de recibir favores, más que de cambiar de vida y costumbres" (ÁVILA, 1962, p. 7). Tras esto, durante un año se preparó el ambiente y cuando se finalizó el primer edificio destinado a la Escuela de orientación, se consiguió que ochenta muchachos se apuntaran para comenzar los cursos.

Las edades estaban comprendidas entre los 11 y los 18 años (ÁVILA, 1962). En 1962 los matriculados eran 350 niños acogidos gratuitamente y repartidos en cinco cursos, con catorce profesores y maestros de taller. Las clases de cultura general se daban por las mañanas y los talleres profesionales se realizaban por la tarde.

La Escuela de Orientación profesional no dependió de forma directa de la comunidad Asuncionista desde un principio, en sus comienzos funcionó bajo la dirección del Ministerio de Educación Nacional que nombró un director y un cuerpo de profesores y maestros, tras unos resultados entendidos como no satisfactorios (ya que los muchachos acudían de manera muy inconsistente) a los dos años la comunidad Asuncionista asumió la dirección de la Escuela.

\section{Talleres}

Como se ha recogido anteriormente, los talleres profesionales se realizaban por la tarde. El ingreso se realizaba a los 12 años y la formación consistía en tres cursos desarrollados durante tres o más años (PÉREZ, 1953) ya que podían repetir algún curso. Los talleres al principio fueron tres, pero a medida que la cobertura económica aumentó, se ampliaron a seis, estos comprendían el desarrollo teórico y práctico de varios oficios: ajuste y mecánica, carpintería, electricidad, imprenta, dibujo industrial y oficinas (RUTE, 1961).

Los niños, a partir del segundo curso y después de haber pasado por los diferentes talleres, se quedaban de forma definitiva en aquel en el que mejor encajaban según sus aptitudes y vocación profesional. Cada aprendiz realizaba un trabajo de examen a modo de resumen sobre los conocimientos incorporados y una aplicación práctica de todo aquello que había aprendido durante el curso, estos trabajos eran posteriormente expuestos.

En cuanto a la labor del maestro de taller, tal como señala Pérez (1953), este únicamente debía orientar el trabajo, ya que se entendía que el estímulo lo llevaba cada 
aprendiz en sí mismo. Todos tenían que realizar el programa que este daba para cada taller al comenzar el curso. Los alumnos más brillantes obtenían al final de curso premios en metálico que oscilaban entre las 200 y las 1000 pesetas, además de tener la opción de ser colocados como oficiales en talleres de la Ciudad de los Muchachos.

Finalizados los tres años y al terminar su formación profesional, los muchachos podían pasar a ser colocados en talleres de Madrid en calidad de oficiales de los distintos trabajos. Por otra parte, tal como señala Pérez (1953), para que su formación religiosa no se redujese, seguían manteniendo contacto con la institución, a la que podían ir todas las noches en calidad de antiguos alumnos (con una asociación propia que se organizó a tal fin), intercambiando impresiones sobre los problemas surgidos en el trabajo y en su vida privada.

\section{Autogobierno cotidiano}

Existía un procedimiento que regulaba la cotidianidad del centro a través de un gobierno participativo que fomentaba la responsabilidad diaria. Todos los alumnos al comienzo del curso y de forma anual elegían democráticamente a aquellos alumnos que pasaban a integrar el denominado Ayuntamiento con el que se repartían diferentes responsabilidades. Este órgano estaba compuesto por Alcalde, Secretario, Juez, Jefe de policía, un Policía por curso, Ministro de portes, Ministro de abastecimiento y uno sin cartera que estaba para sustituir al que faltaba. Todos los componentes de la Alcaldía tenían su responsabilidad controlada por el Alcalde, estando este coordinado desde la Dirección que respondía del proceso. Cada tres meses se renovaban todos los cargos sin perjuicio de poder volver a ser reelegidos sus ocupantes (PÉREZ, 1953).

Tal como se advierte en los documentos, estos cargos venían también a suplir la deficiencia de personal docente con su presencia en talleres, así, en el taller de Dibujo Industrial estaba el alcalde realizando la parte práctica cuando el profesor acababa su explicación y se marchaba a otra clase, en Mecanografía quedaba el Juez, etc.

He aquí el alcalde más joven de la nación.

Es un muchacho simpático y cordial.

¿Contento del cargo?

- Mucho, son excelentes muchachos y casi no hay que castigarlos.

¿Esperabas el nombramiento?

- Fue una sorpresa para mí. Desde entonces he tenido que superarme.

¿Cuántos años tienes?

- Quince y llevo cuarto curso en la ciudad.

Es que ¿eras antes travieso?

- Desde luego, pero he tenido que cambiar. (PÉREZ, 1953, s.p.).

\section{La revista}

El 15 de octubre de 1946 sale a la luz la primera revista mensual titulada Ciudad de los Muchachos, impresa en el taller de imprenta y editada por la Comunidad Asuncionista. Fue el primer órgano de difusión y el primer ingreso con el que se contó para la construcción 
de la Ciudad de los Muchachos. Otras fuentes de ingreso se obtenían a través de campañas de radio donde el Padre Madina explicaba la iniciativa y solicitaba colaboración, la venta de lotería, suscripciones fijas a la obra, ayudas de la institución de la Falange Auxilio Social, particulares, etc. (PÉREZ, 1953). La suscripción mínima en 1960 era de 5 pesetas al mes y la anual de 60 pesetas, muchos suscriptores sobrepasaban esa cuota con creces.

$\mathrm{Su}$ preocupación era la de informar a sus abonados sobre la marcha de la Institución, las mejoras efectuadas, novedades, nuevos materiales, trabajo realizado con los muchachos, etc. En 1953 había 6000 suscriptores, aumentando en 1962 a 18.000. En definitiva, la revista perseguía dos fines: servir de órgano informativo de la Institución y ser vínculo de unión con los suscriptores (PÉREZ, 1953).

La clase de gimnasia ha terminado y marchamos a visitar las diversas instalaciones de que consta el edificio. En primer lugar, la imprenta, montada con arreglo a los más modernos adelantos de la técnica. De ella sale mensualmente la revista "Ciudad de los Muchachos”, que lleva a todos los rincones de España el afán y diario trajín de estas vidas jóvenes y redimidas.

¿Cuántos suscriptores tienen?

- Unos 13.000. Luego hay cuotas desde 200 pesetas hasta 1.

¿Cuántos chicos han pasado por la ciudad?

- Actualmente hay más de 300 exalumnos en contacto directo con nosotros y que trabajan o bien en empresas particulares o en oficinas, y algunos con sueldos muy altos (GARCÍA JÍMENEZ, 1955, p. 5).

\section{Conclusiones}

Bajo la pretensión de educar a la población infanto-juvenil perteneciente a las capas más vulnerables de la población en situación de exclusión o riesgo social, las Boys Town (tanto religiosas como laicas) constituyen un fenómeno socio-educativo que surge para intentar ofrecer respuesta efectiva. Así, se definen como comunidades socio-educativas generalmente alejadas de los grandes núcleos urbanos, donde a través de la formación académica, laboral y moral del niño y mediante un marcado fomento de la responsabilidad personal y social (alcanzado gracias al autogobierno participativo de todo el alumnado), se pretende lograr la reinserción y/o adaptación social.

Dentro del marco teórico y práctico de las Boys Town surge la experiencia socioeducativa de la Ciudad de los Muchachos de Vallecas. Iniciativa que se implementó dentro de uno de los barrios más empobrecidos de Madrid y en uno de los momentos históricos más duros en la historia de España, la posguerra. La naturaleza de su práctica, su fundamentación teórica, su innovadora propuesta metodológica (propia de las Boys Town que se estaban desarrollando en varias partes del mundo en aquella época), unido a las particulares características del lugar y el dramático periodo histórico en el que se desarrolló, hacen de la Ciudad de los Muchachos, como se ha visto, una iniciativa con un gran valor histórico, pedagógico y socio-educativo.

En conclusión, estudiar y reflexionar sobre hitos fundamentales en la historia de la educación favorece la mejora y optimización de los procesos educativos. Así, se enriquece 
el marco teórico y práctico de discusión; se promueve una perspectiva investigadora, justificada, comprometida y crítica sobre los procesos de enseñanza-aprendizaje; se fortalece la mirada sobre las relaciones dialécticas que se establecen entre escuela-docentealumno y contexto social; y, por último, se recuperan debates fundamentales sobre los fines de la educación. El estudio de los primeros años de la Ciudad de los Muchachos permite disponer de una valiosa información que da respuesta a los interrogantes que nos planteamos en un principio, ampliando el conocimiento sobre esta iniciativa pedagógica desde una perspectiva histórica y educativa.

\section{Referencias}

ALONSO-CORTES, María. Problemas pedagógicos de un suburbio madrileño. Madrid: Fondo Documental Romero Marín: Museo de Historia de la Educación "Manuel Bartolomé Cossío": Facultad de Educación: Universidad Complutense de Madrid, 1966.

ANDRÉS-CANDELAS, Mario. La construcción socio-histórica de la "infancia peligrosa" en España. Revista Latinoamericana de Ciencias Sociales, Niñez y Juventud, Manizales, v. 14, n. 1, p. 95-196, 2016.

ARIĖS, Philippe. El niño y la vida familiar en el antiguo régimen. Madrid: Taurus, 1987.

ÁVILA, Inmaculada. La ciudad de los Muchachos. Madrid: Fondo Documental Romero Marín: Museo de Historia de la Educación "Manuel Bartolomé Cossío": Facultad de Educación: Universidad Complutense de Madrid, 1962.

AZAÑA, Manuel. Causas de la guerra de España. Barcelona: Crítica, 1986.

BUENO, Roberto. La situación educativa en Vallecas. Madrid: Fondo Documental Romero Marín: Museo de Historia de la Educación "Manuel Bartolomé Cossío": Facultad de Educación: Universidad Complutense de Madrid, 1963.

CARREÑO, Miryam. Reflexiones sobre el por qué y el para qué de la educación de la "infancia anormal" según el discurso médico-pedagógico español de los inicios del siglo XX. Revista Educación y Pedagogía, Antioquía, v. 17, n. 42, p. 31-44, 2005.

CASCARDI, Michele et al. Where have we been and where are goiong? A conceptual framework for child advocacy. SAGE Open, Thousand Oaks, v. 5, n. 1, p. 1-10, 2015.

CUEVAS, Francisco José. Anarquismo y educación: la propuesta sociopolítica de la pedagogía libertaria. Madrid: Fundación Anselmo Lorenzo, 2003.

DEMAUSE, Lloyd. La evolución de la infancia. In: DEMAUSE, Lloyd (Ed.). Historia de la infancia. Madrid: Alianza, 1982. p. 15-92.

DEWEY, John. Democracia y educación. Madrid: Morata, 1995.

ESPAÑA. Decreto de 17 de mayo de 1940 incluyendo la Institución Libre de Enseñanza entre las entidades reseñadas en el artículo primero del Decreto número 108 de la Junta de Defensa Nacional y disposiciones complementarias. Boletín Oficial del Estado, Madrid, p. 126, 28 mayo 1940. 
FERNÁNDEZ, Matilde. La educación de los excluidos en la Ciudad de los Muchachos: el ejemplo de Vallecas. In: FERNÁNDEZ, Matilde; MÜLLAUER-SEICHTER, Waltraud (Coord.). La integración escolar a debate. Madrid: Pearson Educación, 2009. p. 122-142.

FERNÁNDEZ, Matilde. Vallecas, identidades compartidas, identidades enfrentadas: la ciudad, el pueblo y el campo, el suburbio y el barrio. Revista de Dialectología y Tradiciones Populares, Madrid, v. 62, n. 1, p. 33-83, 2007.

FROUFE QUINTAS, Sindo. Experiencias alternativas singulares. In: ORTEGA ESTABAN, José (Coord.). Educación social especializada. Barcelona: Ariel Educación, 1999. p. 150-164.

GARCÍA JIMÉNEZ, Baldomero. Noticia sobre visita a la Ciudad de los Muchachos de Vallecas. Nueva Alcarria, Guadalajara, p. 5, 26 nov. 1955.

KEY, Ellen. El siglo de los niños. Barcelona: Heinrich, 1906.

KOLTSOV, Mijail. Diario de la guerra de España. Madrid: Backlist, 2009.

LAGO, Regina. Las Repúblicas juveniles. Revista de Pedagogía, Madrid, n. 22, 1931.

LA VANGUARDIA. La rifa de la "Ciudad de los Muchachos". La Vanguardia Española, p. 10, 5 sept. 1951.

MADINA, Luis. Nuevos edificios ampliarán la ciudad de los muchachos de Madrid. ABC, Madrid, p. 33, 12 oct. 1950.

MAKARENKO, Anton Semionovich. Poema pedagógico. Madrid: Akal, 1996.

MAURIIN, Joaquín. Revolución y contrarrevolución en España. París: Ruedo lbérico, 1966.

MELTON, Gary B. Treating children like people: a framework for research and advocacy. Journal of Clinical Child \& Adolescent Psychology, London, v. 34, n. 4, p. 646-657, 2005.

MORROW, Virginia. Invisible children? Toward a reconceptualization of childhood dependency and responsibility. In: JENKS, Chris (Ed.). Childhood: critical concepts in sociology II. Abingdon: Routledge, 2005. p. 246-266.

OSSORIO, Ángel. La España de mi vida: autobiografía. Buenos Aires: Losada, 1941.

PAYÁ, María Raquel. Una ciudad de los muchachos en Valencia. Bordón Revista de la Sociedad Española de Pedagogía, Madrid, n. 15, p. 41-46, 1950.

PÉREZ, María Concepción. Ciudad de los Muchachos. Madrid: Fondo Documental Romero Marín: Museo de Historia de la Educación "Manuel Bartolomé Cossío": Facultad de Educación: Universidad Complutense de Madrid, 1953.

PERICACHO, Francisco Javier. Actualidad de la renovación pedagógica en la Comunidad de Madrid: un estudio a través de escuelas emblemáticas. Evolución y experiencias actuales ante los retos socioeducativos de la sociedad del siglo XXI. Madrid: UCM, 2014. 422 p. Tesis (Doctorado) - Programa Doctorado Conocimiento Pedagógico Avanzado, Universidad Complutense de Madrid, Madrid, 2014a.

PERICACHO, Francisco Javier. Pasado y presente de la renovación pedagógica en España (de finales del Siglo XIX a nuestros días): un recorrido a través de escuelas emblemáticas. Revista Complutense de Educación, Madrid, v. 25, n. 1, p. 47-67, 2014b. 
PLATT, Anthony. Los salvadores del niño o la invención de la delincuencia. México, DF: Siglo XXI, 1988.

PRIETO, Indalecio. Entresijos de la guerra de España. Barcelona: Planeta, 1989.

REILLY, Hugh; WARNEKE, Kevin. Father flanagan of boys town: a man of vision. Crawford: Boys Town Press, 2008.

RODRIGUES-BREITMAN, Miriam. La construcción social de la infancia delincuente. Nueva Sociedad, Buenos Aires, n. 129, p. 152-163, 1994.

RÖHRS, Hermann. Georg Kerschensteiner (1852-1932). Perspectivas, París, n. 3/4, p. 855-872, 1993.

ROUSSEAU, Jean-Jaques. Emilio, o de la educación. Madrid: EDAF, 1982.

RUTE, Rosa. Problemática social y humana de la Ciudad de los Muchachos. Madrid: Fondo documental Romero Marín: Museo de Historia de la Educación "Manuel Bartolomé Cossío", Facultad de Educación: Universidad Complutense de Madrid, 1961.

SAZ, Ismael. Fascismo y franquismo. Valencia: Universitat de València, 2004.

TAGÚEÑ̃, Manuel. Testimonio de dos guerras. Barcelona: Planeta, 2005.

TRILLA, Jaume. Antón Semiónovich Makarenko: la fuerza de la colectividad. In: PEDAGOGÍAS del siglo XX. Barcelona: Ciss Praxis, 1985. p. 95-106.

VIÑAO, Antonio. La alfabetización en España: un proceso cambiante de un mundo multiforme. Revista Electrónica de Educación y Formación Continua de Adultos, Salamanca, v. 3, n. 1, p. 5-19, 2009.

Recibido en: 27.04.2016

Aprobado en: 22.11.2016

Francisco Javier Pericacho Gómez es doctor en Educación por la Universidad Complutense de Madrid (UCM). Ha sido profesor en la Facultad de Formación de Profesorado de la Universidad Autónoma de Madrid (UAM), coordinador del máster universitario en Formación del Profesorado en la Universidad Antonio de Nebrija y profesor invitado en la Universidad de Coimbra (Portugal) y la Regent's University London (Reino Unido). Actualmente es profesor en el Departamento de Educación de la Universidad Antonio de Nebrija.

Mario Andrés-Candelas es máster en Estudios Avanzados en Pedagogía por la Universidad Complutense de Madrid y pedagogo por la misma universidad. Con experiencia en docencia e investigación en Colombia, España y Portugal, actualmente trabaja en el departamento de orientación de la Escuela Ideo (Madrid) y como docente en el Departamento de Didáctica y Organización Escolar de la Facultad de Educación de la Universidad Complutense de Madrid. 